\title{
Basic classes for four-manifolds not of simple type
}

\author{
VICENTE MUÑoZ ${ }^{1}$
}

\begin{abstract}
We extend the notion of basic classes (for the Donaldson invariants) to 4-manifolds with $b^{+}>1$ which are (potentially) not of simple type or satisfy $b_{1}>0$. We also give a structure theorem for the Donaldson invariants of 4-manifolds with $b^{+}>1, b_{1}>0$ and of strong simple type.
\end{abstract}

\section{Introduction.}

The primary purpose of this paper is to extend the notion of basic classes (as defined by Kronheimer and Mrowka [3] for the Donaldson invariants) to all 4-manifolds satisfying $b^{+}>1$. So we drop the conditions $b_{1}=0$ and being of simple type imposed in [3]. We give a (somewhat partial) structure theorem for the Donaldson invariants of any 4-manifold with $b^{+}>1$ (compare [4]).

Donaldson invariants for a (smooth, compact, oriented) 4-manifold $X$ with $b^{+}>1$ (and with a homology orientation) are defined as linear functionals [3]

$$
D_{X}^{w}: \mathbb{A}(X)=\operatorname{Sym}^{*}\left(H_{0}(X) \oplus H_{2}(X)\right) \otimes \Lambda^{*} H_{1}(X) \rightarrow \mathbb{C},
$$

where $w \in H^{2}(X ; \mathbb{Z})$. As for the grading of $\mathbb{A}(X)$, we give degree $4-i$ to the elements in $H_{i}(X)$. If $x \in H_{0}(X)$ denotes the class of a point, we say that $X$ is of $w$-simple type when $D_{X}^{w}\left(\left(x^{2}-4\right) z\right)=0$, for any $z \in \mathbb{A}(X)$. When $X$ has $b_{1}=0$ and it is of $w$-simple type for some $w \in H^{2}(X ; \mathbb{Z})$, then it is of $w^{\prime}$-simple type for any other $w^{\prime} \in H^{2}(X ; \mathbb{Z})$, and it is said to be of simple type for brevity [1]. For 4-manifolds of simple type (with $b^{+}>1$ ), it is customary to define [3] the formal power series (in a variable $t$ ) given by

$$
\mathbb{D}_{X}^{w}(t D)=D_{X}^{w}\left(\left(1+\frac{x}{2}\right) e^{t D}\right)
$$

for any $D \in H_{2}(X)$. We introduce the following notions as in [5]

\footnotetext{
${ }^{1}$ Partially supported by DGES through Spanish Research Project PB97-1095.
} 
Definition 1.1. Let $w \in H^{2}(X ; \mathbb{Z})$. We say that $X$ is of $w$-finite type when there is some $n \geq 0$ such that $D_{X}^{w}\left(\left(x^{2}-4\right)^{n} z\right)=0$, for any $z \in \mathbb{A}(X)$. The order (of $w$-finite type) is the minimum of such $n . X$ is of finite type if it is of $w$-finite type for any $w \in H^{2}(X ; \mathbb{Z})$ and the order (of finite type) is the supremum of all the orders of $w$-finite type of $X$, with $w \in H^{2}(X ; \mathbb{Z})$. Also $X$ is of $w$-strong simple type, for $w \in H^{2}(X ; \mathbb{Z})$, when $D_{X}^{w}\left(\left(x^{2}-\right.\right.$ $4) z)=0$, for any $z \in \mathbb{A}(X)$ and $D_{X}^{w}(\delta z)=0$, for any $\delta \in H_{1}(X)$ and any $z \in \mathbb{A}(X) . X$ is of strong simple type if it is of $w$-strong simple type for any $w \in H^{2}(X ; \mathbb{Z})$.

Clearly strong simple type implies simple type, and the two conditions are equivalent when $b_{1}=0$. Also finite type of order 1 means simple type and order 0 means that the Donaldson invariants are identically zero. Note that by [5, theorem 1.2], all 4-manifolds with $b^{+}>1$ are of finite type. Since $D_{X}^{w+2 \alpha}=(-1)^{\alpha^{2}} D_{X}^{w}$, for any $\alpha \in H^{2}(X ; \mathbb{Z})$, the order of $w$-finite type only depends on $w$ modulo $2 H^{2}(X ; \mathbb{Z})$, and hence the order of finite type is finite. We shall see later (theorem 1.5) that the order of $w$-finite type does not depend on $w \in H^{2}(X ; \mathbb{Z})$ and hence the order of finite type is finite and equal to any order of $w$-finite type.

Our first result is an extension of the structure theorem for the Donaldson invariants as given in [3] [1] to 4-manifolds of strong simple type, in the form that was stated in [5, proposition 9.6] (recall that $Q$ stands for the intersection form).

Theorem 1.2. Let $X$ be a 4-manifold with $b^{+}>1$ and of w-strong simple type for some $w \in H^{2}(X ; \mathbb{Z})$. Then $X$ is of strong simple type and $\mathbb{D}_{X}^{w}=e^{Q / 2} \sum(-1)^{\frac{K_{i} \cdot w+w^{2}}{2}} a_{i} e^{K_{i}}$, for finitely many $K_{i} \in H^{2}(X ; \mathbb{Z})$ and rational numbers $a_{i}$ (the collection is empty when the invariants all vanish). These classes are lifts to integral cohomology of $w_{2}(X)$. Moreover, for any embedded surface $S \hookrightarrow X$ of genus $g$, representing a non-torsion homology class and with $S^{2} \geq 0$, one has $2 g-2 \geq S^{2}+\left|K_{i} \cdot S\right|$, for all $K_{i}$.

When we put $b_{1}=0$ we recover the structure theorem of Kronheimer and Mrowka given in [3] for the Donaldson invariants of 4-manifolds of simple type with $b_{1}=0$ and $b^{+}>1$ (see also [1] for the case of simply connected 4-manifolds). It is possible that the proof provided in [3] can be adapted to prove theorem 1.2, but there are difficulties as one of the starting conditions for the analysis in [3] is that of admissibility in [3, definition 2.23], which can not be removed in general. A. Stipsicz informed the author that he encountered this same problem in the computation of the Donaldson invari- 
ants of the 4-torus [6]. We have decided to include a self-contained proof of theorem 1.2 as it is used by the author in the determination of the Donaldson invariants of the product of two compact surfaces of genus bigger than one in [5, subsection 9.3]. It will also serve to clarify the ideas behind the proof of our more general structure theorem for the Donaldson invariants of arbitrary 4-manifolds with $b^{+}>1$ (theorem 1.6).

Definition 1.3. If $X$ is a 4-manifold with $b^{+}>1$ and of strong simple type, then the basic classes of $X$ will be the cohomology classes $K_{i}$ of theorem 1.2 such that $a_{i} \neq 0$.

Remark 1.4. There are many 4-manifolds with $b_{1}>0$ and $b^{+}>1$ which are of strong simple type. For instance, $X=\Sigma_{g} \times \Sigma_{h}$, where $\Sigma_{r}$ is a compact surface of genus $r$ and $g, h \geq 1$, are of strong simple type, as it is proved in [5, theorem 1.4]. On the other hand, there are also 4-manifolds with $b^{+}>1$ not of strong simple type. If $X$ is any 4-manifold with $b^{+}>1$ and non-vanishing Donaldson invariants, then $X^{\prime}=X \# \mathbb{S}^{1} \times \mathbb{S}^{3}$ is not of strong simple type, as follows from [5, lemma 6.4]. Nonetheless for $X$ of strong simple type and $X^{\prime}=X \# \mathbb{S}^{1} \times \mathbb{S}^{3}$, let $\delta$ be the image in $H_{1}\left(X^{\prime}\right)$ of the generator of $\pi_{1}\left(\mathbb{S}^{1} \times \mathbb{S}^{3}\right)$. We have

$$
D_{X^{\prime}}^{w}\left(\delta\left(1+\frac{x}{2}\right) e^{t D}\right)=D_{X}^{w}\left(\left(1+\frac{x}{2}\right) e^{t D}\right)=\mathbb{D}_{X}^{w}(t D),
$$

which has the same shape as that provided by theorem 1.2 .

For general 4-manifolds with $b^{+}>1$ it is not licit to suppose that $\left(x^{2}-4\right)$ and all $\delta \in H_{1}(X)$ kill the Donaldson invariants. In principle, there might be 4-manifolds with $b_{1}=0, b^{+}>1$ not of simple type [4] (although they have not been found so far) and, in any case, remark 1.4 tells us that there are examples where the 1-homology classes act non-trivially. We have the following two main results:

Theorem 1.5. Let $X$ be a 4-manifold with $b^{+}>1$. Let $w, w^{\prime} \in H^{2}(X ; \mathbb{Z})$. Then the order of $w$-finite type and the order of $w^{\prime}$-finite type of $X$ are equal. This number is thus the order of finite type of $X$.

Theorem 1.6. Let $X$ be a 4-manifold with $b^{+}>1, w \in H^{2}(X ; \mathbb{Z})$ and $z \in \Lambda^{*} H_{1}(X) \subset \mathbb{A}(X)$ an homogeneous element. Then there are finitely many cohomology classes $K_{i} \in H^{2}(X ; \mathbb{Z})$ and non-zero polynomials $p_{i}, q_{i} \in$ 
$\operatorname{Sym}^{*} H^{2}(X) \otimes \mathbb{Q}[\lambda]$ such that

$$
\begin{aligned}
D_{X}^{w}\left(z e^{t D+\lambda x}\right)= & e^{Q(t D) / 2+2 \lambda} \sum p_{i}(t D, \lambda) e^{K_{i} \cdot t D} \\
& +e^{-Q(t D) / 2-2 \lambda} \sum q_{i}(t D, \lambda) e^{\mathbf{i} K_{i} \cdot t D},
\end{aligned}
$$

for any $D \in H_{2}(X)$. The collection of classes $K_{i}$ is independent of $w$ and $K_{i}$ are lifts to integral cohomology of $w_{2}(X)$.

Remark 1.7. Let $d_{0}=-w^{2}-\frac{3}{2}\left(1-b_{1}+b^{+}\right)$and $d=\operatorname{deg}(z) / 2$. Here $d_{0}-d \in$ $\mathbb{Z}$. Then $D_{X}^{w}\left(z e^{\mathrm{i} t D-\lambda x}\right)=\mathbf{i}^{d_{0}-d} D_{X}^{w}\left(z e^{t D+\lambda x}\right), \mathbf{i}=\sqrt{-1}$, so $q_{i}(t D, \lambda)=$ $\mathbf{i}^{d-d_{0}} p_{i}(\mathbf{i} t D,-\lambda)$ for all $i$. Therefore the $p_{i}$ determine the $q_{i}$ and conversely. Also if $K_{j}=-K_{i}$ then $p_{j}(-t D, \lambda)=(-1)^{d_{0}-d} p_{i}(t D, \lambda)$. So the classes $K_{i}$ come actually in pairs $\pm K_{i}$.

We finally have the following definition, which agrees with definition 1.3 for strong simple type manifolds.

Definition 1.8. Let $X$ be a 4 -manifold with $b^{+}>1$ and $z \in \Lambda^{*} H_{1}(X)$ an homogeneous element. The cohomology classes $K_{i}$ of theorem 1.6 such that $p_{i} \neq 0$ are called basic classes for $(X, z)$. The union of all the basic classes for $(X, z)$ where $z$ runs through any homogeneous basis of $\Lambda^{*} H_{1}(X)$ is the set of basic classes for $X$.

The proof of theorem 1.6 is based on two techniques. On the one hand, the Fukaya-Floer homology of the three-manifold $Y=\Sigma \times \mathbb{S}^{1}$, where $\Sigma$ is a compact surface, as determined in [5], which we recall in section 2 for the convenience of the reader. On the other hand, partial use of the blow-up formula in [2] which relates the Donaldson invariants of a 4-manifold $X$ and those of its blow-up $\tilde{X}=X \# \overline{\mathbb{C P}}^{2}$. Section 3 is devoted to prove theorem 1.2 and in section 4 we study the Donaldson invariants of general 4-manifolds with $b^{+}>1$ in order to prove theorems 1.5 and 1.6.

\section{Fukaya-Floer homology revisited.}

Let $Y$ be a 3-manifold with $b_{1}>0$ and $w \in H^{2}(Y ; \mathbb{Z} / 2 \mathbb{Z})$ non-zero. For any loop $\delta \subset Y$, we have defined the Fukaya-Floer homology [5]

$$
H F F_{*}(Y, \delta),
$$

which is a $\mathbb{C}[[t]]$-module, endowed with a $\mathbb{C}[[t]]$-bilinear pairing

$$
\langle,\rangle: H F F_{*}(Y, \delta) \otimes H F F_{*}(-Y,-\delta) \rightarrow \mathbb{C}[[t]],
$$


where $-Y$ is $Y$ with reversed orientation. For every 4-manifold $X_{1}$ with boundary $\partial X_{1}=Y$ and $w_{1} \in H^{2}(X ; \mathbb{Z})$ such that $\left.w_{1}\right|_{Y}=w \in H^{2}(Y ; \mathbb{Z} / 2 \mathbb{Z})$, $z \in \mathbb{A}\left(X_{1}\right)$ and $D_{1} \subset X_{1}$ a 2-cycle with $\partial D_{1}=\delta$, one has a relative invariant

$$
\phi^{w}\left(X_{1}, e^{t D_{1}}\right) \in H F F_{*}(Y, \delta) .
$$

The relevant gluing theorem is:

Theorem $2.1([5])$. Let $X=X_{1} \cup_{Y} X_{2}$ and $w \in H^{2}(X ; \mathbb{Z})$ such that there exists $\Sigma \in H^{2}(X ; \mathbb{Z})$ whose Poincaré dual lies in the image of $H_{2}(Y ; \mathbb{Z}) \rightarrow$ $H_{2}(X ; \mathbb{Z})$, and satisfies $w \cdot \Sigma \equiv 1(\bmod 2):$ Put $w_{i}=\left.w\right|_{X_{i}} \in H^{2}\left(X_{i} ; \mathbb{Z}\right)$. Let $D \in H_{2}(X)$ decomposed as $D=D_{1}+D_{2}$ with $D_{i} \subset X_{i}, i=1,2$, 2-cycles with $\partial D_{1}=\delta, \partial D_{2}=-\delta$. Choose $z_{i} \in \mathbb{A}\left(X_{i}\right), i=1,2$. Then

$$
D_{X}^{(w, \Sigma)}\left(z_{1} z_{2} e^{t D}\right)=\left\langle\phi^{w_{1}}\left(X_{1}, z_{1} e^{t D_{1}}\right), \phi^{w_{2}}\left(X_{2}, z_{2} e^{t D_{2}}\right)\right\rangle,
$$

where $D_{X}^{(w, \Sigma)}=D_{X}^{w}+D_{X}^{w+\Sigma}$. When $b^{+}=1$ the invariants are calculated for metrics on $X$ giving a long neck.

We restrict to the case $Y=\Sigma \times \mathbb{S}^{1}$, where $\Sigma$ is a surface of genus $g \geq 1$, $w=$ P.D. $\left[\mathbb{S}^{1}\right], \delta=\mathbb{S}^{1} \subset Y$. As $Y \cong(-Y)$, we have a natural identification $H F F_{*}\left(Y, \mathbb{S}^{1}\right) \cong H F F^{*}\left(Y, \mathbb{S}^{1}\right)$ and a pairing

$$
\langle,\rangle: H F F^{*}\left(Y, \mathbb{S}^{1}\right) \otimes H F F^{*}\left(Y, \mathbb{S}^{1}\right) \rightarrow \mathbb{C}[[t]] .
$$

Let $A=\Sigma \times D^{2}$ be the product of $\Sigma$ times a 2-dimensional disc and consider the horizontal section $\Delta=\Sigma \times$ pt $\subset A$. Let $\left\{\gamma_{i}\right\}$ be a symplectic basis for $H_{1}(\Sigma)$ with $\gamma_{i} \cdot \gamma_{g+i}=1,1 \leq i \leq g$. The Fukaya-Floer homology $H F F^{*}\left(Y, \mathbb{S}^{1}\right)$ is actually a $\mathbb{C}[[t]]$-algebra $[5$, section 5$]$ generated by $\alpha=$ $2 \phi^{w}\left(A, \Sigma e^{t \Delta}\right), \beta=-4 \phi^{w}\left(A, x e^{t \Delta}\right)$ and $\psi_{i}=\phi^{w}\left(A, \gamma_{i} e^{t \Delta}\right), 1 \leq i \leq 2 g$, where the product is determined by $\phi^{w}\left(A, z_{1} e^{t \Delta}\right) \phi^{w}\left(A, z_{2} e^{t \Delta}\right)=\phi^{w}\left(A, z_{1} z_{2} e^{t \Delta}\right)$, $z_{1}, z_{2} \in \mathbb{A}(\Sigma)$. In particular $\phi^{w}\left(A, z e^{t \Delta}\right), z \in \mathbb{A}(\Sigma)$, generate $H F F^{*}\left(Y, \mathbb{S}^{1}\right)$. The mapping class group of $\Sigma$ acts on $H F F^{*}\left(Y, \mathbb{S}^{1}\right)$ factoring through an action of the symplectic group $\operatorname{Sp}(2 g, \mathbb{Z})$ on $\left\{\psi_{i}\right\}$. The invariant part is generated by $\alpha, \beta$ and $\gamma=-2 \sum \psi_{i} \psi_{g+i}$.

In general, we shall have the following situation: $X$ is a 4-manifold with $b^{+}>1$ and $\Sigma \hookrightarrow X$ is an embedded surface of genus $g \geq 1$ and self-intersection zero such that there exists $w \in H^{2}(X ; \mathbb{Z})$ with $w \cdot \Sigma \equiv 1$ $(\bmod 2)$. Let $A$ be a small tubular neighbourhood of $\Sigma$ in $X$ and denote by $X_{1}$ the complement of the interior of $A$, so that $X_{1}$ is a 4-manifold with boundary $\partial X_{1}=Y=\Sigma \times \mathbb{S}^{1}$ and $X=X_{1} \cup_{Y} A$. Take $D \in H_{2}(X)$ with 
$D \cdot \Sigma=1$. Then $D$ can be represented by a cycle $D=D_{1}+\Delta$, where $D_{1} \subset X_{1}, \partial D_{1}=\mathbb{S}^{1}$ and $\Delta=\mathrm{pt} \times D^{2} \subset A$. Theorem 2.1 says

$$
D_{X}^{(w, \Sigma)}\left(z e^{t D+s \Sigma+\lambda x}\right)=\left\langle\phi^{w}\left(X_{1}, z e^{t D_{1}+s \Sigma+\lambda x}\right), \phi^{w}\left(A, e^{t \Delta}\right)\right\rangle,
$$

for any $z \in \mathbb{A}\left(X_{1}\right)$. Here $t$ is a formal variable, and both terms are meant to be developed in powers of $s$ and $\lambda$. Also $\Sigma$ denotes the homology class represented by the surface and the corresponding cohomology class by Poincaré duality. The effective Fukaya-Floer homology, $\widehat{H F F}_{g}^{*}$, (see [5, definition 7.1]) is the sub- $\mathbb{C}[[t]]$-module of $H F F_{g}^{*}$ with the property that

$$
\phi^{w}\left(X_{1}, z e^{t D_{1}}\right) \in \widetilde{H F F_{g}^{*}} \subset H F F_{g}^{*} .
$$

If $R$ is a polynomial such that $R(\alpha, \beta)=0$ acting on $\widetilde{H F F}_{g}^{*}$ then

$$
R\left(2 \frac{\partial}{\partial s},-4 \frac{\partial}{\partial \lambda}\right) \phi^{w}\left(X_{1}, z e^{t D_{1}+s \Sigma+\lambda x}\right)=0,
$$

and therefore $R\left(2 \frac{\partial}{\partial s},-4 \frac{\partial}{\partial \lambda}\right) D_{X}^{(w, \Sigma)}\left(z e^{t D+s \Sigma+\lambda x}\right)=0$. The Donaldson invariants $D_{X}^{(w, \Sigma)}$ satisfy thus partial differential equations coming from the polynomials vanishing on $\widetilde{H F F_{g}^{*}}$. We have the following result

Proposition 2.2 ([5, theorem 7.2]). The effective Fukaya-Floer homology is a sub-C $\mathbb{C}[t]]$-module $\widetilde{H F F_{g}^{*}} \subset H F F_{g}^{*}$ such that there is a direct sum decomposition

$$
\widetilde{H F F_{g}^{*}}=\bigoplus_{r=-(g-1)}^{g-1} R_{g, r},
$$

where $R_{g, r}$ are free $\mathbb{C}[[t]]$-modules such that, in $R_{g, r}, \alpha-(4 r \mathbf{i}-2 t), \beta-8$ and $\gamma$ are nilpotent if $r$ is even, $\alpha-(4 r+2 t), \beta+8$ and $\gamma$ are nilpotent if $r$ is odd.

\section{Strong simple type manifolds.}

This section is devoted to the proof of theorem 1.2. We start with two technical lemmas. We shall abbreviate strong simple type to "sst" in this section.

Lemma 3.1. Let $X$ be a 4-manifold with $b^{+}>1$ and $w, \Sigma \in H^{2}(X ; \mathbb{Z})$ such that $\Sigma^{2}=0$ and $w \cdot \Sigma \equiv 1(\bmod 2)$. If $X$ is both of $w$-sst and of $(w+\Sigma)$-sst 
and $D \in H_{2}(X)$ then there exist power series $f_{r, D}(t),-(g-1) \leq r \leq g-1$, such that

$$
\mathbb{D}_{X}^{w}(t D+s \Sigma)=e^{Q(t D+s \Sigma) / 2} \sum_{r=-(g-1)}^{g-1} f_{r, D}(t) e^{2 r s}
$$

i.e. $\mathbb{D}_{X}^{w}(t D+s \Sigma)$ is a solution of the differential equation

$$
\prod_{r=-(g-1)}^{g-1}\left(\frac{\partial}{\partial s}-(2 r+t(D \cdot \Sigma))\right)
$$

Moreover

$$
\mathbb{D}_{X}^{w+\Sigma}(t D+s \Sigma)=e^{Q(t D+s \Sigma) / 2} \sum_{r=-(g-1)}^{g-1}(-1)^{r+1} f_{r, D}(t) e^{2 r s} .
$$

Proof. It is enough to prove (3.1) for $D \in H_{2}(X)$ with $D \cdot \Sigma=1$, using linearity and continuity. Consider an embedded surface $\Sigma \hookrightarrow X$ of genus $g \geq 1$ representing the Poincare dual of $\Sigma \in H^{2}(X ; \mathbb{Z})$. Then we are in the situation described in section 2 and will stick to the notations used therein. The relative Donaldson invariant

$$
\phi^{w}\left(X_{1}, e^{t D_{1}}\right) \in H F F_{*}\left(\Sigma \times \mathbb{S}^{1}, \mathbb{S}^{1}\right)
$$

lies in the kernels of $\beta^{2}-64$ and all $\psi_{i}, 1 \leq i \leq 2 g$. This is clear as, for instance,

$$
\left\langle\left(\beta^{2}-64\right) \phi^{w}\left(X_{1}, e^{t D_{1}}\right), \phi^{w}\left(A, z e^{t \Delta}\right)\right\rangle=D_{X}^{(w, \Sigma)}\left(16\left(x^{2}-4\right) z e^{t D}\right)=0,
$$

for any $\phi^{w}\left(A, z e^{t \Delta}\right)$, since $X$ is both of $w$-sst and of $(w+\Sigma)$-sst. Thus $\left(\beta^{2}-64\right) \phi^{w}\left(X_{1}, e^{t D_{1}}\right)=0$.

Thus in order to compute

$$
D_{X}^{(w, \Sigma)}\left(e^{t D+s \Sigma+\lambda x}\right)=\left\langle\phi^{w}\left(X_{1}, e^{t D_{1}}\right), \phi^{w}\left(A, e^{t \Delta+s \Sigma+\lambda x}\right)\right\rangle,
$$

it only matters the projection of $\phi^{w}\left(A, e^{t \Delta+s \Sigma+\lambda x}\right)$ to the reduced FukayaFloer homology (see [5, definition 6.1])

$$
{\overline{H F F_{g}}}_{g}^{*}=H F F_{g}^{*} /\left(\beta^{2}-64, \psi_{1}, \ldots, \psi_{2 g}\right) H F F_{g}^{*} .
$$


By [5, theorem 6.2],

$$
\overline{H F F}_{g}^{*}=\bigoplus_{r=-(g-1)}^{g-1} \bar{R}_{g, r}
$$

where

$$
\bar{R}_{g, r}= \begin{cases}\mathbb{C}[[t]][\alpha, \beta] /(\alpha-(4 r \mathbf{i}-2 t), \beta-8) & r \text { even } \\ \mathbb{C}[[t]][\alpha, \beta] /(\alpha-(4 r+2 t), \beta+8) & r \text { odd }\end{cases}
$$

Now we translate the relations of $\overline{H F F}_{g}^{*}$ into partial differential equations satisfied by the Donaldson invariants. For instance, the relation

$$
(\beta-8) \prod_{\substack{-(g-1) \leq r \leq g-1 \\ r \text { odd }}}(\alpha-(4 r+2 t))=0
$$

gives the differential equation

$$
\left(\frac{\partial}{\partial \lambda}+2\right) \prod_{\substack{-(g-1) \leq r \leq g-1 \\ r \text { odd }}}\left(\frac{\partial}{\partial s}-(2 r+t)\right) D_{X}^{(w, \Sigma)}\left(e^{t D+s \Sigma+\lambda x}\right)=0 .
$$

This finally yields the existence of power series $g_{r}(t),-(g-1) \leq r \leq g-1$ (we drop the $D$ from the subindex), such that

$$
D_{X}^{(w, \Sigma)}\left(e^{t D+s \Sigma+\lambda x}\right)=\sum_{r \text { odd }} g_{r}(t) e^{s t+2 r s+2 \lambda}+\sum_{r \text { even }} g_{r}(t) e^{-s t+2 r i s-2 \lambda} .
$$

If $d_{0}=d_{0}(w)=-w^{2}-\frac{3}{2}\left(1-b_{1}+b^{+}\right)$denotes half the dimensions (modulo 4) of the moduli spaces of anti-self-dual connections of $S O(3)$-bundles determined by $w$, then

$$
\begin{aligned}
D_{X}^{w}\left(e^{t D+s \Sigma+\lambda x}\right)= & \frac{1}{2}\left(\sum_{r \text { odd }} g_{r}(t) e^{s t+2 r s+2 \lambda}+\sum_{r \text { even }} g_{r}(t) e^{-s t+2 r \mathbf{i} s-2 \lambda}+\right. \\
& \left.+\sum_{r \text { odd }} \mathbf{i}^{-d_{0}} g_{r}(\mathbf{i} t) e^{-s t+2 r i s-2 \lambda}+\sum_{r \text { even }} \mathbf{i}^{-d_{0}} g_{r}(\mathbf{i} t) e^{s t-2 r s+2 \lambda}\right)
\end{aligned}
$$

and hence

$$
\mathbb{D}_{X}^{w}(t D+s \Sigma)=\sum f_{r}(t) e^{s t+2 r s}
$$

where

$$
f_{r}(t)= \begin{cases}g_{r}(t) & r \text { odd } \\ \mathbf{i}^{-d_{0}} g_{-r}(\mathrm{i} t) & r \text { even }\end{cases}
$$


We leave $\mathbb{D}_{X}^{w+\Sigma}$ to the reader upon noting that $d_{0}(w+\Sigma) \equiv d_{0}(w)+2$ $(\bmod 4)$.

Lemma 3.2. In the situation of lemma $3.1, X$ is of $w$-sst if and only if it is of $(w+\Sigma)$-sst.

Proof. Arguing by contradiction, suppose $X$ is of $w$-sst but not of $(w+\Sigma)$ sst. Then there exists $z=\left(x^{2}-4\right)^{r} \delta_{1} \cdots \delta_{p}$ with $\delta_{i} \in H_{1}(X)$ and $r+p>0$ such that $D_{X}^{w}\left(z e^{t D+s \Sigma+\lambda x}\right)=0, D_{X}^{w+\Sigma}\left(z e^{t D+s \Sigma+\lambda x}\right)$ is non-zero but

$$
D_{X}^{w+\Sigma}\left(z\left(x^{2}-4\right) e^{t D+s \Sigma+\lambda x}\right)=0
$$

and $D_{X}^{w+\Sigma}\left(z \delta e^{t D+s \Sigma+\lambda x}\right)=0$ for any $\delta \in H_{1}(\Sigma)$. Keeping the notations of the proof of the previous lemma, $\phi^{w}\left(X_{1}, z e^{t D_{1}}\right)$ lies in the kernel of $\beta^{2}-64$ and $\gamma$ and so, arguing as in lemma 3.1 , it is

$$
D_{X}^{(w, \Sigma)}\left(z e^{t D+s \Sigma+\lambda x}\right)=\sum_{r \text { odd }} g_{r}(t) e^{s t+2 r s+2 \lambda}+\sum_{r \text { even }} g_{r}(t) e^{-s t+2 r \mathbf{i} s-2 \lambda},
$$

for some power series $g_{r}(t),-(g-1) \leq r \leq g-1$. In particular $D_{X}^{w}\left(z e^{t D+s \Sigma+\lambda x}\right)$ is non-zero, so $X$ is not of $w$-sst. This contradiction proves the lemma.

Proof of theorem 1.2. Now we proceed to the proof of theorem 1.2 by steps.

Step 1. $X$ is of strong simple type.

Let $S$ be a 4 -manifold with $b^{+}>1$ and $w \in H^{2}(S ; \mathbb{Z})$. Let $\tilde{S}=S \# \overline{\mathbb{C P}}^{2}$ denote its blow-up and let $E$ stand for the cohomology class of the exceptional divisor. Therefore $H^{2}(\tilde{S} ; \mathbb{Z})=H^{2}(S ; \mathbb{Z}) \oplus \mathbb{Z} E$. The general blow-up formula [2] implies that $S$ is of $w$-sst if and only if $\tilde{S}$ is of $w$-sst if and only if $\tilde{S}$ is of $(w+E)$-sst.

With this said, suppose $X$ is of $w$-sst for some $w \in H^{2}(X ; \mathbb{Z})$. We shall prove that $X$ is of $w^{\prime}$-sst for any other $w^{\prime} \in H^{2}(X ; \mathbb{Z})$. Consider any cohomology class $L \in H^{2}(X ; \mathbb{Z})$ with $N=L^{2}>0$. Blow up $X$ at $N$ points to obtain $\tilde{X}=X \# N \overline{\mathbb{C P}}^{2}$, with $E_{1}, \ldots, E_{N}$ denoting the exceptional divisors. Let $L^{\prime}=L-E_{1}-\ldots-E_{N} \in H^{2}(\tilde{X} ; \mathbb{Z})$ which has $\left(L^{\prime}\right)^{2}=0$. As $X$ is of $w$-sst, $\tilde{X}$ will be both of $w$-sst and $\left(w+E_{1}\right)$-sst (recall that $N>0)$. Now $w \cdot L^{\prime}$ and $\left(w+E_{1}\right) \cdot L^{\prime}$ have different parity since $E_{1} \cdot L^{\prime}=1$. Therefore one of them is odd. If $w \cdot L^{\prime} \equiv 1(\bmod 2)$, then lemma 3.2 implies 
that $\tilde{X}$ is of $\left(w+L^{\prime}\right)=\left(w+L-E_{1}-\ldots-E_{N}\right)$-sst, and hence $X$ is of $(w+L)$-sst. Alternatively, if $\left(w+E_{1}\right) \cdot L^{\prime} \equiv 1(\bmod 2)$, then $\tilde{X}$ is of $\left(w+E_{1}+L^{\prime}\right)=\left(w+L-E_{2}-\ldots-E_{N}\right)$-sst and $X$ of $(w+L)$-sst again. In conclusion, $X$ is of $(w+L)$-sst, for any $L \in H^{2}(X ; \mathbb{Z})$ with $L^{2}>0$.

Now given $w$ and $w^{\prime}$, take $T \in H^{2}(X ; \mathbb{Z})$ with $T^{2}>0$. For $n$ large, it will be $\left(w^{\prime}-w+n T\right)^{2}>0$. Considering $L=w^{\prime}-w+n T$, it follows that $X$ is of $(w+L)=\left(w^{\prime}+n T\right)$-sst. Now taking $L=-n T$, we have that $X$ is of $w^{\prime}$-sst, as required.

Step 2. There exists at least one $w \in H^{2}(X ; \mathbb{Z})$ with

$$
\mathbb{D}_{X}^{w}=e^{Q / 2} \sum a_{i, w} e^{K_{i}}
$$

for finitely many cohomology classes $K_{i} \in H^{2}(X ; \mathbb{Z})$ and rational numbers $a_{i, w}$.

Let $S$ be a 4-manifold with $b^{+}>1$ and of sst. If $\tilde{S}$ is the blow-up of $S$ with exceptional divisor $E$, then the blow-up formula [2] says that $\mathbb{D}_{S}^{w}(t D)=\mathbb{D}_{\tilde{S}}^{w}(t D)$ and $\mathbb{D}_{S}^{w}(t D)=\left.\frac{\partial}{\partial r}\right|_{r=0} \mathbb{D}_{\tilde{S}}^{w+E}(t D+r E)$, for $D \in H_{2}(X)$, so we see that it is enough to prove the claim for $\tilde{S}$.

After possibly blowing up, we can suppose that $X$ has an indefinite odd intersection form of the form $Q=r(1) \oplus s(-1)$, with $r, s \geq 2, n=r+s$. Put $A_{1}, \ldots, A_{r}, B_{1}, \ldots, B_{s}$ for the corresponding basis. Then we set $\Sigma_{1}=$ $A_{2}-B_{1}, \Sigma_{j}=A_{j}+B_{1}, 2 \leq j \leq r, \Sigma_{r+1}=B_{2}-A_{1}, \Sigma_{r+j}=B_{j}+A_{1}$, $2 \leq j \leq s$, and $w=A_{1}+B_{1}$. Then we have a subgroup

$$
H=\left\langle\Sigma_{1}, \ldots, \Sigma_{n}\right\rangle \subset \bar{H}_{2}(X ; \mathbb{Z})=H_{2}(X ; \mathbb{Z}) / \text { torsion, }
$$

such that $2 \bar{H}_{2}(X ; \mathbb{Z}) \subset H$, with $\Sigma_{j}^{2}=0$ and $w \cdot \Sigma_{j} \equiv 1(\bmod 2), 1 \leq j \leq n$.

We represent $\Sigma_{j}$ by embedded surfaces of genus $g_{j} \geq 1$. Lemma 3.1 implies then

$$
\begin{aligned}
& \mathbb{D}_{X}^{w}\left(t_{1} \Sigma_{1}+\cdots+t_{n} \Sigma_{n}\right) \\
& \quad=e^{Q\left(t_{1} \Sigma_{1}+\cdots+t_{n} \Sigma_{n}\right) / 2} \sum_{\substack{-\left(g_{j}-1\right) \leq r_{j} \leq g_{j}-1 \\
1 \leq j \leq n}} a_{r_{1} \ldots r_{n}, w} e^{2 r_{1} t_{1}+\cdots+2 r_{n} t_{n}}
\end{aligned}
$$

for complex numbers $a_{r_{1} \ldots r_{n}, w}$. They are rational because of the rationality of the Donaldson invariants. The claim follows. $K_{i}$ are integral cohomology classes since $2 \bar{H}_{2}(X ; \mathbb{Z}) \subset H$.

Step 3. $\quad K_{i}$ are lifts of $w_{2}(X)$ to integer coefficients. 
Equivalently, we need to prove that $K_{i} \cdot x \equiv x^{2}(\bmod 2)$, for any $x \in$ $\bar{H}_{2}(X ; \mathbb{Z})$. Clearly formula (3.2) implies that $K_{i} \cdot \Sigma_{j} \equiv 0=\Sigma_{j}^{2}(\bmod 2)$, for $1 \leq j \leq n$. Take $x \in \bar{H}_{2}(X ; \mathbb{Z})-H$. There is some index $k$ such that $x \cdot \Sigma_{k} \neq 0$. We can find an integer $m$ such that $x^{\prime}=x+m \Sigma_{k}$ has $N=\left(x^{\prime}\right)^{2} \geq 0$ and $w \cdot x^{\prime} \equiv 1(\bmod 2)\left(\operatorname{recall}\right.$ that $w \cdot \Sigma_{j} \equiv 1(\bmod 2)$ for all $j$ ). We blow up $X$ at $N$ points to get $\tilde{X}=X \# N \overline{\mathbb{C P}}^{2}$ with exceptional divisors $E_{1}, \ldots, E_{N}$. Then $y=x^{\prime}-E_{1}-\ldots-E_{N}$ has $y^{2}=0$ and $w \cdot y \equiv 1$ $(\bmod 2)$. The blow-up formula [2] says

$$
\mathbb{D}_{\tilde{X}}^{w}=\mathbb{D}_{X}^{w} \cdot e^{-\left(E_{1}^{2}+\cdots+E_{N}^{2}\right) / 2} \cosh E_{1} \cdots \cosh E_{N}=e^{Q / 2} \sum \frac{a_{i, w}}{2^{N}} e^{K_{i}+\sum \pm E_{l}},
$$

so the basic classes of $\tilde{X}$ are of the form $K_{i}+\sum \pm E_{l}$, with $K_{i}$ basic classes for $X$. Now lemma 3.1 applied to $w$ and $y$ implies in particular that

$$
0 \equiv\left(K_{i}+\sum \pm E_{l}\right) \cdot y \equiv K_{i} \cdot x^{\prime}+N \quad(\bmod 2)
$$

and hence $K_{i} \cdot x \equiv x^{2}(\bmod 2)$, for all $K_{i}$.

Step 4. $\quad \mathbb{D}_{X}^{w^{\prime}}=e^{Q / 2} \sum(-1)^{\frac{K_{i} \cdot w^{\prime}+w^{\prime 2}}{2}} a_{i} e^{K_{i}}$, for any other $w^{\prime} \in H^{2}(X ; \mathbb{Z})$, where we put $a_{i}=(-1)^{-\frac{K_{i} \cdot w+w^{2}}{2}} a_{i, w}$ (by step 3 the exponent is an integer).

Lemma 3.1 implies that if we have proved the claim for $w^{\prime}$ and $w^{\prime} \cdot \Sigma \equiv 1$ $(\bmod 2)$ and $\Sigma^{2}=0$, then the claim is true for $w^{\prime}+\Sigma$. Now to prove the assertion for any $w^{\prime} \in H^{2}(X ; \mathbb{Z})$, it is enough to consider $w^{\prime}=w+L$, with $N=L^{2}>0$ as in step 1 . Keep those notations and suppose for instance that we are in the case $\left(w+E_{1}\right) \cdot L^{\prime} \equiv 1(\bmod 2)$ (i.e. $w \cdot L$ is even). Then the blow-up formula again says

$$
\mathbb{D}_{\tilde{X}}^{w+E_{1}}=-\mathbb{D}_{X}^{w} \cdot e^{-\left(E_{1}^{2}+\cdots+E_{N}^{2}\right) / 2} \sinh E_{1} \cosh E_{2} \cdots \cosh E_{N},
$$

so the coefficient of the basic class $K_{i}+\sum a_{l} E_{l}$, where $\left(a_{l}\right)_{l=1}^{N}$ is a sequence of numbers $a_{l}= \pm 1$, is $(-1)^{\frac{a_{1}+1}{2}} a_{i, w} / 2^{N}$. By lemma 3.1,

$$
\mathbb{D}_{\tilde{X}}^{w+L-E_{2}-\ldots-E_{N}}=e^{Q / 2} \sum c_{K_{i}+\sum a_{l} E_{l}}\left(a_{i, w} / 2^{N}\right) e^{K_{i}+\sum a_{l} E_{l}},
$$

where

$$
\begin{aligned}
c_{K_{i}+\sum a_{l} E_{l}} & =(-1)^{\frac{a_{1}+1}{2}}(-1)^{\frac{\left(K_{i}+\sum a_{l} E_{l}\right) \cdot L^{\prime}}{2}}+1 \\
& =(-1)^{\frac{K_{i} \cdot L+L^{2}}{2}}(-1)^{\frac{a_{2}+1}{2}} \cdots(-1)^{\frac{a_{N}+1}{2}}(-1)^{N-1} .
\end{aligned}
$$


Using the blow-up formula again together with the standard fact $D_{X}^{w+2 \alpha}=$ $(-1)^{\alpha^{2}} D_{X}^{w}$, we get $\mathbb{D}_{X}^{w+L}=e^{Q / 2} \sum(-1)^{\frac{K_{i} \cdot L+2 w \cdot L+L^{2}}{2}} a_{i, w} e^{K_{i}}$ (since $w \cdot L$ is even), as required.

Step 5. The final assertion is proved as follows. Let $S \hookrightarrow X$ be an embedded surface of genus $g$ with $N=S^{2} \geq 0$ and representing a nontorsion homology class. The argument in [3, page 709] reduces to prove only the case $N>0$. If the genus is $g=0$ then the Donaldson invariants vanish identically and hence it is trivially true. In the case $g \geq 1$, blow-up $X$ at $N$ points to get $\tilde{X}=X \# N \overline{\mathbb{C P}}^{2}$ with exceptional divisors $E_{1}, \ldots, E_{N}$. Consider the proper transform $\tilde{S}$ of $S$ which is an embedded surface in $\tilde{X}$ of genus $g$ representing the homology class $S-E_{1}-\ldots-E_{N}$. Then lemma 3.1 applied to $\tilde{X}, \tilde{S}$ and $w=E_{1}$ gives

$$
2(g-1) \geq\left|\left(K_{i}+\sum \pm E_{l}\right) \cdot\left(S-E_{1}-\ldots-E_{N}\right)\right|
$$

for all $K_{i}$ basic classes of $X$. Therefore we have $2 g-2 \geq\left|K_{i} \cdot S\right|+S^{2}$.

\section{Basic classes.}

We first prove theorem 1.5. We start with the following analogue of lemma 3.2.

Lemma 4.1. In the situation of lemma 3.1, $X$ is of $w$-finite type of order $k$ if and only if it is of $(w+\Sigma)$-finite type of order $k$.

Proof. Arguing by contradiction, suppose $X$ is of $w$-finite type of order $k$ and of $(w+\Sigma)$-finite type of order $k+a, a>0$. Then there exists $z=\left(x^{2}-\right.$ $4)^{k+a-1} \delta_{1} \cdots \delta_{p}$ with $\delta_{i} \in H_{1}(X), p \geq 0$, such that $D_{X}^{w}\left(z e^{t D+s \Sigma+\lambda x}\right)=0$, $D_{X}^{w+\Sigma}\left(z e^{t D+s \Sigma+\lambda x}\right)$ is non-zero but $D_{X}^{w+\Sigma}\left(z\left(x^{2}-4\right) e^{t D+s \Sigma+\lambda x}\right)=0$ and $D_{X}^{w+\Sigma}\left(z \delta e^{t D+s \Sigma+\lambda x}\right)=0$, for any $\delta \in H_{1}(\Sigma)$. The arguments in the proof of lemma 3.2 now carry over verbatim.

Proof of theorem 1.5. It goes as in step 1 of the proof of theorem 1.2 with the difference that we use lemma 4.1 and note the following: Let $S$ be a 4-manifold with $b^{+}>1$ and $w \in H^{2}(S ; \mathbb{Z})$. Let $\tilde{S}=S \# \overline{\mathbb{C P}}^{2}$ denote its blow-up and let $E$ stand for the cohomology class of the exceptional divisor. 
Then $S$ is of $w$-finite type of order $k$ if and only if $\tilde{S}$ is of $w$-finite type of order $k$ if and only if $\tilde{S}$ is of $(w+E)$-finite type of order $k$.

Proof of theorem 1.6. Now we proceed to the proof of theorem 1.6. The analogue of lemma 3.1 is the following:

Lemma 4.2. Let $X$ be a 4-manifold with $b^{+}>1$ and $w, \Sigma \in H^{2}(X ; \mathbb{Z})$ such that $\Sigma^{2}=0$ and $w \cdot \Sigma \equiv 1(\bmod 2)$. Let $z \in \Lambda^{*} H_{1}(X)$ homogeneous. Take $D \in H_{2}(X)$. Then

$$
\begin{aligned}
D_{X}^{w}\left(z e^{t D+s \Sigma+\lambda x}\right)= & e^{Q(t D+s \Sigma) / 2+2 \lambda} \sum_{r=-(g-1)}^{g-1} P_{r}(t, s, \lambda) \\
& +e^{-Q(t D+s \Sigma) / 2-2 \lambda} \sum_{r=-(g-1)}^{g-1} Q_{r}(t, s, \lambda),
\end{aligned}
$$

where $P_{r}(t, s, \lambda)$ is a solution of the differential equations $\left(\frac{\partial}{\partial s}-2 r\right)^{N}$, $\left(\frac{\partial}{\partial \lambda}\right)^{N}$, and $Q_{r}(t, s, \lambda)$ is a solution of $\left(\frac{\partial}{\partial s}-2 r \mathbf{i}\right)^{N},\left(\frac{\partial}{\partial \lambda}\right)^{N}$, for $N$ sufficiently large. Moreover

$$
\begin{aligned}
D_{X}^{w+\Sigma}\left(z e^{t D+s \Sigma+\lambda x}\right)= & e^{Q(t D+s \Sigma) / 2+2 \lambda} \sum_{r=-(g-1)}^{g-1}(-1)^{r+1} P_{r}(t, s, \lambda)+ \\
& +e^{-Q(t D+s \Sigma) / 2-2 \lambda} \sum_{r=-(g-1)}^{g-1}(-1)^{r} Q_{r}(t, s, \lambda) .
\end{aligned}
$$

Proof. We proceed as in the proof of lemma 3.1, but in this case we have $\phi^{w}\left(X_{1}, z e^{t D_{1}+s \Sigma+\lambda x}\right) \in \widetilde{H F F_{g}^{*}} \subset H F F_{g}^{*}$. Now the eigenvalues of $(\alpha, \beta, \gamma)$ in $\widetilde{H F F}_{g}^{*}$ given by proposition 2.2 yield that

$$
\begin{aligned}
D_{X}^{(w, \Sigma)}\left(z e^{t D+s \Sigma+\lambda x}\right)= & e^{Q(t D+s \Sigma) / 2+2 \lambda} \sum_{-(g-1) \leq r \leq g-1} P_{r}(t, s, \lambda) \\
& +e^{-Q(t D+s \Sigma) / 2-2 \lambda} \sum_{\substack{r \text { odd } \\
-(g-1) \leq r \leq g-1 \\
r \text { even }}} Q_{r}(t, s, \lambda),
\end{aligned}
$$


where $P_{r}$ and $Q_{r}$ satisfy the differential equations in the statement. Then put $d_{0}=d_{0}(w)=-w^{2}-\frac{3}{2}\left(1-b_{1}+b^{+}\right)$, so

$$
\begin{aligned}
& D_{X}^{w}\left(e^{t D+s \Sigma+\lambda x}\right) \\
& =\frac{1}{2}\left(e^{Q(t D+s \Sigma) / 2+2 \lambda}\left(\sum_{\substack{g-1) \leq r \leq g-1 \\
r \text { odd }}} P_{r}(t, s, \lambda)+\mathbf{i}^{-d_{0}} \sum_{\substack{-(g-1) \leq r \leq g-1 \\
r \text { even }}} Q_{r}(\mathbf{i} t, \mathbf{i} s,-\lambda)\right)\right.
\end{aligned}
$$

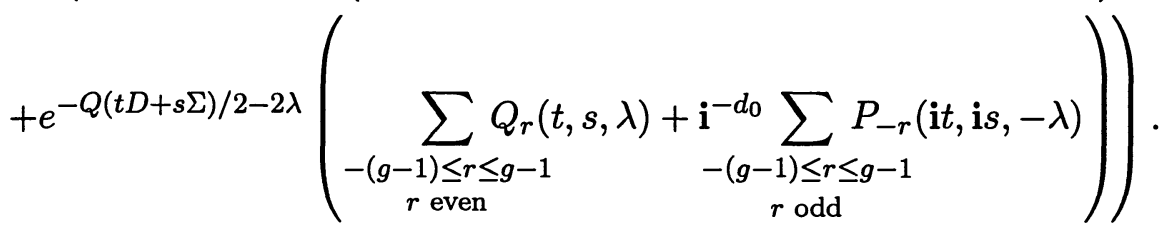

We leave $D_{X}^{w+\Sigma}$ to the reader.

Step 1. Suppose $X$ is a 4 -manifold with $b^{+}>1, w \in H^{2}(X ; \mathbb{Z}), z \in$ $\Lambda^{*} H_{1}(X)$ homogeneous and that there is a subgroup $H=\left\langle\Sigma_{1}, \ldots, \Sigma_{n}\right\rangle \subset$ $\bar{H}_{2}(X ; \mathbb{Z})$ such that $2 \bar{H}_{2}(X ; \mathbb{Z}) \subset H$, with $\Sigma_{j}^{2}=0$ and $w \cdot \Sigma_{j} \equiv 1(\bmod 2)$, $1 \leq j \leq n$. Then (1.1) holds.

We represent $\Sigma_{j}$ by embedded surfaces of genus $g_{j} \geq 1$. Lemma 4.2 . implies that

$$
\begin{aligned}
& D_{X}^{w}\left(z e^{t_{1} \Sigma_{1}+\cdots+t_{n} \Sigma_{n}+\lambda x}\right) \\
= & e^{Q\left(t_{1} \Sigma_{1}+\cdots+t_{n} \Sigma_{n}\right) / 2+2 \lambda} \sum_{-\left(g_{j}-1\right) \leq r_{j} \leq g_{j}-1} P_{r_{1} \ldots r_{n}, w}\left(s_{1}, \ldots, s_{n}, \lambda\right) e^{2 r_{1} t_{1}+\cdots+2 r_{n} t_{n}} \\
+e^{-Q\left(t_{1} \Sigma_{1}+\cdots+t_{n} \Sigma_{n}\right) / 2-2 \lambda} \sum_{-\left(g_{j}-1\right) \leq r_{j} \leq g_{j}-1} Q_{r_{1} \ldots r_{n}, w}\left(s_{1}, \ldots, s_{n}, \lambda\right) e^{\mathrm{i}\left(2 r_{1} t_{1}+\cdots+2 r_{n} t_{n}\right)}, &
\end{aligned}
$$

where $P$ 's and $Q$ 's are polynomials. This finishes the step, noting again that $K_{i}$ are integral cohomology classes since $2 \bar{H}_{2}(X ; \mathbb{Z}) \subset H$.

Step 2. Fix an arbitrary $w \in H^{2}(X ; \mathbb{Z})$. Then (1.1) holds for $X$ and $w$.

First note that if $w, \Sigma \in H^{2}(X ; \mathbb{Z})$ with $w \cdot \Sigma \equiv 1(\bmod 2)$ and $\Sigma^{2}=0$ then (1.1) holds for $w$ implies (1.1) holds for $w+\Sigma$.

The relationship of the Donaldson invariants of a 4-manifold $S$ and the blow-up $\tilde{S}=S \# \overline{\mathbb{C P}}^{2}$ is not as straightforward as in the strong simple type 
case, therefore the strategy of proving that (1.1) holds for one $w \in H^{2}(X ; \mathbb{Z})$ implies that it holds for any other $w^{\prime} \in H^{2}(X ; \mathbb{Z})$ by blowing-up does not work easily. Instead we fix $w \in H^{2}(X ; \mathbb{Z})$ and shall look for a blow-up $\tilde{X}=X \# m \overline{\mathbb{C P}}^{2}$ with exceptional divisors $E_{1}, \ldots, E_{m}$ such that there exists $\tilde{w} \in H^{2}(\tilde{X} ; \mathbb{Z})$ of the form $\tilde{w}=w+\sum a_{i} E_{i}, a_{i}$ integers, with $(\tilde{X}, \tilde{w})$ satisfying the conditions in step 1. This is enough since $D_{X}^{w}\left(z e^{t D+\lambda x}\right)=D_{\tilde{X}}^{w}\left(z e^{t D+\lambda x}\right)$ and $D_{X}^{w}\left(z e^{t D+\lambda x}\right)=\left.\frac{\partial}{\partial r}\right|_{r=0} D_{\tilde{X}}^{w+E}\left(z e^{t D+r E+\lambda x}\right)$, for $D \in H_{2}(X)$.

We can blow up to ensure that $Q=r(1) \oplus s(-1), r \geq 2, s \geq 1$, with corresponding basis $A_{1}, \ldots, A_{r}, B_{1}, \ldots, B_{s}$ and $w \cdot B_{n} \equiv 0(\bmod 2)$. There are several cases:

- Suppose that (up to reordering) $w \cdot A_{1} \equiv 1(\bmod 2), w \cdot A_{2} \equiv 0$ $(\bmod 2)$. Then blow up once, $\tilde{X}=X \# \overline{\mathbb{C P}}^{2}$, with $E$ the exceptional divisor. Set

$$
\begin{aligned}
\Sigma_{j} & =\left\{\begin{array}{lll}
A_{j}+E & \text { if } w \cdot A_{j} \equiv 0 & (\bmod 2) \\
A_{j}+B_{n} & \text { if } w \cdot A_{j} \equiv 1 & (\bmod 2)
\end{array}\right. \\
\Sigma_{r+j} & =\left\{\begin{array}{lll}
B_{j}-A_{1} & \text { if } w \cdot B_{j} \equiv 0 & (\bmod 2) \\
B_{j}-A_{2} & \text { if } w \cdot B_{j} \equiv 1 & (\bmod 2)
\end{array}\right. \\
\Sigma_{n+1} & =E-A_{2} .
\end{aligned}
$$

Then the subgroup $H=\left\langle\Sigma_{1}, \ldots, \Sigma_{n+1}\right\rangle \subset H_{2}(\tilde{X} ; \mathbb{Z})$ and $\tilde{w}=w+E$ satisfy the required properties in step 1.

- Suppose that $w \cdot A_{j} \equiv 1(\bmod 2)$, for all $j$. Then put $\Sigma=A_{1}+B_{n}$ and $w^{\prime}=w+\Sigma$. Then $w^{\prime}$ satisfies the conditions of the previous case so (1.1) holds for $w^{\prime}$. Now $w^{\prime} \cdot \Sigma \equiv 1(\bmod 2)$ and $\Sigma^{2}=0$, so (1.1) also holds for $w$.

- Suppose that $w \cdot A_{j} \equiv 0(\bmod 2)$, for all $j$ and that there exists $B_{1}$ such that $w \cdot B_{1} \equiv 1(\bmod 2)$. Then put $\Sigma=A_{1}+B_{1}$ and $w^{\prime}=w+\Sigma$, and work as in the previous case.

- Suppose $w \equiv 0(\bmod 2)$. Blow-up once and put $\tilde{w}=w+E$. It reduces to the previous case.

Before carrying on with the proof of theorem 1.6, let us pause to give a characterization of the basic classes of $X$. So far we only can say that the basic classes are relative to a particular $w \in H^{2}(X ; \mathbb{Z})$. So we define a basic class for $(X, z, w)$ to be a cohomology class $K_{i}$ provided by step 2 , such that $p_{i} \neq 0$. 
Proposition 4.3. Let $X$ be a 4-manifold with $b^{+}>1, z \in \Lambda^{*} H_{1}(X)$ homogeneous and $w \in H^{2}(X ; \mathbb{Z})$. Then $K \in H^{2}(X ; \mathbb{Z})$ is a basic class for $(X, z, w)$ if and only if there exists $z^{\prime} \in \mathbb{A}_{\text {even }}(X)=\operatorname{Sym}^{*}\left(H_{0}(X) \oplus H_{2}(X)\right)$ such that $D_{X}^{w}\left(z z^{\prime} e^{t D+\lambda x}\right)=e^{Q(t D) / 2+2 \lambda+K \cdot t D}$.

Proof. Take $N$ larger than the order of finite type of $X$. Then

$$
D_{X}^{w}\left(z\left(1+\frac{x}{2}\right)^{N} e^{t D+\lambda x}\right)=e^{Q(t D) / 2+2 \lambda} \sum p_{i}(t D, \lambda) e^{K_{i} \cdot t D} .
$$

Consider a basis $\left\{D_{1}, \ldots, D_{n}\right\}$ of $\bar{H}_{2}(X ; \mathbb{Z})$. Let $\alpha_{j}=K \cdot D_{j}, j=1, \ldots, n$. Then take

$$
z^{\prime}=\prod_{j} \prod_{\beta_{j} \neq \alpha_{j}}\left(D_{j}-\beta_{j}\right)^{N}
$$

for a large enough $N$ (just take it larger than the degrees of the polynomials $\left.p_{i}\right)$. Then $D_{X}^{w}\left(z\left(1+\frac{x}{2}\right)^{N} z^{\prime} e^{t D+\lambda x}\right)=e^{Q(t D) / 2+2 \lambda} p(t D, \lambda) e^{K \cdot t D}$, for a polynomial $p$. Take some $z^{\prime \prime}=c\left(1-\frac{x}{2}\right)^{m}\left(D_{1}-\alpha_{1}\right)^{m_{1}} \cdots\left(D_{n}-\alpha_{n}\right)^{m_{n}}$, for appropriate exponents and constant $c$, to get

$$
D_{X}^{w}\left(z\left(1+\frac{x}{2}\right)^{N} z^{\prime} z^{\prime \prime} e^{t D+\lambda x}\right)=e^{Q(t D) / 2+2 \lambda} e^{K \cdot t D} .
$$

The converse is obvious.

We leave the following characterization of basic classes in terms of the blow-up to the reader.

Corollary 4.4. Let $X$ be a 4-manifold with $b^{+}>1, z \in \Lambda^{*} H_{1}(X)$ homogeneous and $w \in H^{2}(X ; \mathbb{Z})$. Let $\tilde{X}=X \# \overline{\mathbb{C P}}^{2}$ be its blow-up with exceptional divisor $E$. Then $K \in H^{2}(X ; \mathbb{Z})$ is a basic class for $(X, z, w)$ if and only if there exists $z^{\prime} \in \mathbb{A}_{\text {even }}(X)$ such that

$$
D_{\tilde{X}}^{w}\left(z z^{\prime} e^{t D+\lambda x}\right)=e^{Q(t D) / 2+2 \lambda+K \cdot t D} \cosh (E \cdot t D)
$$

if and only if there exists $z^{\prime} \in \mathbb{A}_{\text {even }}(X)$ such that

$$
D_{\tilde{X}}^{w+E}\left(z z^{\prime} e^{t D+\lambda x}\right)=-e^{Q(t D) / 2+2 \lambda+K \cdot t D} \sinh (E \cdot t D) .
$$

Now we resume the proof of theorem 1.6.

Step 3. The basic classes of $(X, z)$ are independent of $w \in H^{2}(X ; \mathbb{Z})$. 
By lemma 4.2 the basic classes for $(X, z, w)$ and $(X, z, w+\Sigma)$ are the same for any $\Sigma$ such that $\Sigma^{2}=0$ and $w \cdot \Sigma \equiv 1(\bmod 2)$. The argument runs as in step 4 of section 3 using the characterization of basic classes gathered in corollary 4.4. We need to use lemma 4.2 in the blow-up manifold $\tilde{X}$ with an extra $z^{\prime} \in \mathbb{A}_{\text {even }}(\Sigma)$, but it is easy to see that its statement still holds.

Step 4. Any basic class $K_{i}$ is a lift of $w_{2}(X)$ to integer coefficients.

This is as in step 3 of section 3 by noting that corollary 4.4 implies in particular that $K \pm E$ are basic classes of the blow-up of $X$.

Note also the following corollary to corollary 4.4

Corollary 4.5. If $K \in H^{2}(X ; \mathbb{Z})$ is a basic class for $X$ then $K \pm E$ are basic classes for the blow-up $\tilde{X}=X \# \overline{\mathbb{C P}}^{2}$.

Remark 4.6. It is natural to expect that there are more basic classes $K+$ $m E, m$ odd, $m \neq \pm 1$.

Acknowledgements. Thanks to Ignasi Mundet for comments on early versions of this manuscript. I am grateful to the Department of Mathematics of Universidad Autónoma de Madrid for their hospitality.

\section{References.}

[1] R. Fintushel and R.J. Stern, Donaldson invariants of 4-manifolds with simple type, Jour. Diff. Geometry, 42 (1995), 577-633.

[2] R. Fintushel and R.J. Stern, The blow-up formula for Donaldson invariants, Annals of Math. 143 (1996), 529-546.

[3] P.B. Kronheimer and T.S. Mrowka, Embedded surfaces and the structure of Donaldson's polynomial invariants, Jour. Diff. Geometry, 41 (1995), 573-734.

[4] P.B. Kronheimer and T.S. Mrowka, The structure of Donaldson's invariants for four-manifolds not of simple type, http://www.math.harvard.edu/HTML/Individuals/Peter_Kronheimer.html

[5] V. Muñoz, Fukaya-Floer homology of $\Sigma \times \mathbb{S}^{1}$ and applications, Jour. Diff. Geometry, to appear.

[6] A. Stipsicz, Computations of Donaldson invariants via cut and paste techniques, Ph. D. Thesis, Rutgers University, NJ, 1994. 
Facultad de Ciencias

UNIVERSIDAD DE MÁLAGA

29071 MÁLAGA

SPAIN

E-mail address: vmunoz@agt.cie.uma.es

RECeIVed November 17, 1998. 